\title{
Comparing the resolution of Bartlett and MVDR estimators for bottom parameter estimation using pressure and vector sensor short array data
}

\author{
P. Felisberto, J. Schneiderwind, P. Santos, O. Rodriguez, S.M. Jesus \\ LARSyS,University of Algarve, Faro, Portugal \\ Email: pfelis@ualg.pt
}

\begin{abstract}
This work compares the resolution of a pressure and vector sensor based conventional Bartlett estimator, with their MVDR estimator counterparts, in the context of bottom characterization with a short vertical array. Santos et al. [1] demonstrated the gain of a vector sensor array (VSA) based linear estimator (Bartlett) for generic parameter estimation. Moreover, it was shown that for bottom characterization the highest resolution of the estimates were achieved with the vertical particle velocity measurements alone. The present work highlights the gain in parameter resolution of a VSA based MVDR estimator. It is shown, that also for a MVDR estimator, the vector sensor array data improves the resolution of parameter estimation. But, it is also shown, through simulations, that for bottom parameter estimation, the pressure based MVDR estimator has higher resolution and sidelobe attenuation than the VSA based Bartlett estimator. These results were verified for experimental data acquired by a four element, $30 \mathrm{~cm}$ long vertical VSA in the 8-14 kHz band, during the Makai Experiment 2005 sea trial, off Kauai I., Hawaii (USA).

Index Terms-Vector sensor arrays, bottom parameter estimation, Bartlett estimator, MVDR estimator.
\end{abstract}

\section{INTRODUCTION}

Vector sensors, which measure the particle velocity in addition to pressure, have been widely used for source localization either as linear array ([2] and references within) or as single device [3]. The particle velocity is a vectorial field given by the gradient of the pressure. Therefore vector sensors exhibit intrinsic spatial filtering capabilities, that for example allow vector sensor arrays to overcome the left-right ambiguity problem of linear hydrophone arrays. In the last decade, vector sensors have been also introduced in other underwater acoustic applications such as port and waterway security [4], underwater communications [5] and geoacoustic inversion [1], [6], [7]. State-of-the-art vector sensor devices are low size, operate over wide frequency bands of few $\mathrm{Hz}$ to several $\mathrm{kHz}$ and have large dynamic ranges [8]. Santos et al. [1] used data acquired during the MAKAI'05 experiment [9] by a vertical array of 4 such vector sensors (VSA), $30 \mathrm{~cm}$ aperture to perform geoacoustic parameter estimation with signals in the ten kilohertz band. Although, the high frequency of the signals used, the few elements and the small aperture of the VSA, it was possible to estimate the sediment velocity, density and attenuation in line with the ground truth for the area. The estimates were obtained using an extension of the Bartlett estimator to include particle velocity measurements. It was shown that the VSA measurements remarkably improve the resolution of the estimates, when compared with pressure only data of an array of similar characteristics (number of sensors, array aperture). Moreover, the highest resolution of the bottom parameters was achieved when the vertical component of the particle velocity was considered alone. Since, state-of-the-art VSA are defence sensitive devices that are not commercially available, one can consider the usage of a vertical array, which elements are pairs of closely located hydrophones to explore the vertical particle velocity for geoacoustic parameter estimation. Felisberto at al. [10] using a normal mode representation of the particle velocity field discussed the potential gain provided by particle velocity measurements and settle rules to design a measurement system (frequency, source depth, sensor separation) for geoacoustic parameter estimation based on vector sensors (VS) or alternatively in pairs of closely located hydrophones. It was shown for the noise free case that the performance achieved by vertical particle velocity sensors or pair of hydrophones are similar, when hydrophone separation obeys the sampling rules applied to high order modal functions. In the presence of surface ambient noise, simulated using the model proposed by Kuperman [11], the performance of the Bartlett estimator based on the hydrophone pairs slightly degraded, but in the analyzed cases they perform generally better than using hydrophone arrays directly (i.e. without estimating the vertical particle velocity field). These studies give rise to the development of an array of 4 hydrophone pairs for bottom characterization that is functionally equivalent to the vector sensor array described above. The array named Short Hydrophone Array ${ }^{1}$ (SHA), was tested in an acoustic tank and will be used in a forthcoming sea trial to characterize sea bottom covered by seagrasses [12]. Although the tests performed so far shown that the expected functionality of SHA was achieved at least in controlled conditions, the implementation of the hydrophone pairs imposed more stringent requirements to hydrophones (size, phase characteristics and its tolerance, directivity patterns) and respective signal conditioning electronics than these encountered in conventional hydrophone arrays.

In order to achieve a higher resolution of the estimates a number of so called high resolution estimators have been

\footnotetext{
${ }^{1}$ www.siplab.fct.ualg.pt/equipment.shtml
} 
proposed [13]. Such estimators have been applied with vector sensor arrays for direction of arrival estimation [14], [15]. In the present work one discusses the application of the well known high resolution Minimum Variance Distortionless Response (MVDR) estimator to bottom characterization with short array and high frequency signals. One compares through simulations the resolution of the estimates obtained from a pressure only (conventional hydrophone array) and vertical particle velocity using the Bartlett and the MVDR estimators. The results show that vertical particle velocity based estimators present higher resolution and side lobe attenuation than their pressure counterparts, but the pressure based MVDR has resolution and side lobe attenuation similar to that of Bartlett vertical particle velocity based estimator. Despite the high frequency signals used the simulation results were verified for experimental data acquired during the Makai'05 sea trial.

This paper is organized as follows: Section II reviews the vector sensor measurement model and the VSA-based Bartlett estimator proposed by Santos et al [1]. It is derived the VSAbased MVDR estimator for generic parametric estimation. The pressure and vertical particle velocity based estimators are applied for seabed parameter estimation in simulated context. Section III presents the experimental results of seabed parameters estimates using high frequency signals. Finally, Section IV draws some conclusions.

\section{THEORETICAL FRAMEWORK}

In underwater acoustics very often the parameter estimation problem is solved by Matched Field Inversion (MFI). The basic principle of MFI is simple: find a replica - a solution of the direct problem given a set of parameters, that best matches the measured data. The match between the data and the replica is evaluated by a cost function, also known as processor or estimator. The estimator correlates the measured data (typically pressure data) with the replica, maximizing the output power for a given input signal [13]. Hereafter, it is assumed that one has at hand a convenient propagation model to compute the replica. Next, following Santos et al. [1] it is reviewed the particle velocity-pressure joint model and the VSA based Bartlett estimator. Then, it is introduced the VSA based MVDR estimator and the performance of the various estimators are compared through simulations.

\section{A. Measurement model}

A vector sensor measures the pressure $p$ and the particle velocity components $\mathbf{v}=\left[v_{x}, v_{y}, v_{z}\right]$. For a monochromatic signal at frequency $\omega$, the field due to a signal $s$ with power $\sigma_{s}^{2}$, measured at an $L$-element VSA, can be written as [1]

$$
\left[\begin{array}{l}
\mathbf{y}_{p}\left(\boldsymbol{\Theta}_{\mathbf{0}}\right) \\
\mathbf{y}_{v}\left(\boldsymbol{\Theta}_{\mathbf{0}}\right)
\end{array}\right]=\left[\begin{array}{c}
1 \\
\mathbf{u}\left(\boldsymbol{\Theta}_{\mathbf{0}}\right)
\end{array}\right] \otimes \mathbf{h}\left(\boldsymbol{\Theta}_{\mathbf{0}}\right) s+\left[\begin{array}{l}
\mathbf{n}_{p} \\
\mathbf{n}_{v}
\end{array}\right]
$$

where $\Theta_{0}$ is a set of environmental parameters, $\otimes$ is the Kronecker product, $\mathbf{h}\left(\boldsymbol{\Theta}_{\mathbf{0}}\right)$ is the channel frequency response (pressure), $\mathbf{u}=\left[u_{x}, u_{y}, u_{z}\right]$ is a unity vector giving the direction of the particle velocity field, $\mathbf{n}_{p}, \mathbf{n}_{\mathbf{v}}$, are additive, zero mean and white noise components, with variance $\sigma_{n}^{2}$, uncorrelated between each sensor and their components, and uncorrelated with the signal.

\section{B. Data correlation matrix}

The structure of the data correlation matrix, hereafter referred to as correlation matrix, is a key point on the estimator design and its performance analysis.

Considering data model (1) and that signal and all noise components are uncorrelated, the pressure-particle velocity correlation matrix can by written as

$$
\mathbf{R}_{p v}\left(\Theta_{0}\right)=\left[\begin{array}{cc}
\mathbf{R}_{p}\left(\Theta_{0}\right) & \mathbf{0}_{L \times 3 L} \\
\mathbf{0}_{3 L \times L} & \mathbf{R}_{v}\left(\Theta_{0}\right)
\end{array}\right]
$$

where $\mathbf{0}_{L \times 3 L}$ and $\mathbf{0}_{3 L \times L}$ are zeros matrices of dimension $L \times 3 L$ and $3 L \times L$, respectively. $\mathbf{R}_{p}\left(\Theta_{0}\right)$ and $\mathbf{R}_{v}\left(\Theta_{0}\right)$ are the pressure and particle velocity correlation matrices.

The pressure, particle velocity and pressure-particle velocity correlation matrices are given by

$$
\begin{aligned}
& \mathbf{R}_{p}\left(\Theta_{0}\right)=\mathbf{h}\left(\Theta_{0}\right) \mathbf{h}^{H}\left(\Theta_{0}\right) \sigma_{s}^{2}+\sigma_{n}^{2} \mathbf{I}_{L} \\
& \mathbf{R}_{v}\left(\Theta_{0}\right)=\left[\mathbf{u}\left(\Theta_{0}\right) \otimes \mathbf{h}\left(\Theta_{0}\right)\right]\left[\mathbf{u}\left(\Theta_{0}\right) \otimes \mathbf{h}\left(\Theta_{0}\right)\right]^{H} \sigma_{s}^{2}+\sigma_{n}^{2} \mathbf{I}_{3 L}
\end{aligned}
$$

$\mathbf{R}_{p v}\left(\Theta_{0}\right)=\left[\mathbf{u}_{e}\left(\Theta_{0}\right) \otimes \mathbf{h}\left(\Theta_{0}\right)\right]\left[\mathbf{u}_{e}\left(\Theta_{0}\right) \otimes \mathbf{h}\left(\Theta_{0}\right)\right]^{H} \sigma_{s}^{2}+\sigma_{n}^{2} \mathbf{I}_{4 L}$,

where $\mathbf{I}_{N}, N=\{L, 3 L, 4 L\}$, is the identity matrix of dimension $N \times N$ and $\mathbf{u}_{e}\left(\Theta_{0}\right)=\left[\begin{array}{c}1 \\ \mathbf{u}\left(\Theta_{0}\right)\end{array}\right]$ is the extended direction vector. Next, without loss of generality it is assumed that $\left\|\mathbf{h}\left(\Theta_{0}\right)\right\|=1$.

\section{VSA-Bartlett estimator}

Following [1] the pressure and particle velocity Bartlett estimators, respectively $P_{p}^{B}(\boldsymbol{\Theta})$ and $P_{v}^{B}(\boldsymbol{\Theta})$, are given by

$$
\begin{aligned}
& P_{p}^{B}(\boldsymbol{\Theta})=B_{p}(\Theta) \sigma_{s}^{2}+\sigma_{n}^{2}, \\
& P_{v}^{B}(\boldsymbol{\Theta})=\left|\mathbf{u}^{H}(\Theta) \mathbf{u}\left(\Theta_{0}\right)\right|^{2} B_{p}(\Theta)+\sigma_{n}^{2},
\end{aligned}
$$

where $\Theta$ represent the set of parameters and $B_{p}(\Theta)=$ $\left|\mathbf{h}^{H}(\Theta) \mathbf{h}(\Theta)\right|^{2}$ is the noise free Bartlett power. The particle velocity Bartlett estimator is proportional to the pressure counterpart. The weight $\left|\mathbf{u}^{H}(\Theta) \mathbf{u}\left(\Theta_{0}\right)\right|^{2}$ represent a directivity factor that improves the estimator resolution and reduces side lobes [1].

\section{VSA-MVDR estimator}

The well known MVDR estimator is a solution of a constrained optimization problem, which idea is to suppress all components in the data that do not resemble the replica [16]. When compared with Bartlett, the MVDR estimator presents narrower main lobe, therefore higher resolution, and improved side lobe cancelation. Generically, MVDR estimator power $P^{C}(\Theta)$ is given by

$$
P^{C}(\Theta)=\left[\mathbf{m}^{H}(\Theta) \mathbf{R}^{-1} \mathbf{m}(\Theta)\right]^{-1},
$$

where $\mathbf{R}$ is the data correlation matrix and $\mathbf{m}(\Theta)$ is the replica for a given set of parameters $\Theta$. 
Considering the structure of the pressure and particle velocity correlation matrices, respectively (3) and (4), and the results presented in [16] page 360 and [1], it is straightforward to demonstrate that the pressure $P_{p}^{C}(\boldsymbol{\Theta})$ and particle velocity $P_{v}^{C}(\boldsymbol{\Theta})$ MVDR estimator power are given by

$P_{p}^{C}(\boldsymbol{\Theta})=\sigma_{n}^{2}\left[L-\frac{\sigma_{s}^{2}\left|\mathbf{h}^{H}(\boldsymbol{\Theta}) \mathbf{h}\left(\boldsymbol{\Theta}_{0}\right)\right|^{2}}{L \sigma_{s}^{2}+\sigma_{n}^{2}}\right]^{-1}$,

$P_{v}^{C}(\boldsymbol{\Theta})=\sigma_{n}^{2}\left[3 L-\frac{\sigma_{s}^{2}\left|\mathbf{u}^{H}(\boldsymbol{\Theta}) \mathbf{u}\left(\boldsymbol{\Theta}_{\mathbf{0}}\right)\right|^{2}\left|\mathbf{h}^{H}(\boldsymbol{\Theta}) \mathbf{h}\left(\boldsymbol{\Theta}_{\mathbf{0}}\right)\right|^{2}}{3 L \sigma_{s}^{2}+\sigma_{n}^{2}}\right]^{-1}$.

One can notice that the greater the term in brackets the smaller the estimator power, which minimum $\sigma_{n}^{2} / L$ for the pressure case and $\sigma_{n}^{2} / 3 L$ for the particle velocity is attained when the inner product between replica and data are 0 (replica and data are orthogonal). The directivity factor $\left|\mathbf{u}^{H}(\boldsymbol{\Theta}) \mathbf{u}\left(\boldsymbol{\Theta}_{0}\right)\right|^{2}$ in the particle velocity estimator, which represent the square of the inner product between the replica unity vector $\mathbf{u}^{H}(\boldsymbol{\Theta})$ and the data unity factor $\mathbf{u}^{H}\left(\boldsymbol{\Theta}_{0}\right)$, gives rise to an improved side lobe attenuation of the particle velocity estimator when compared to its pressure counterpart.
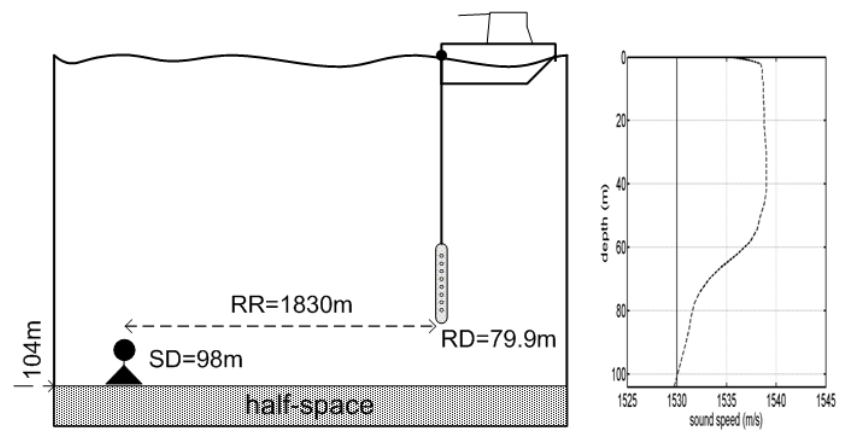

Fig. 1. Makai'05 baseline environment: geometry (left), sound speed profile (dashed line, right)

\section{E. Simulation results}

In this section a simulation scenario based on Makai'05 experimental setup [1] is considered. Results on experimental data will be presented in Section III.

The environment is a range independent $104 \mathrm{~m}$ water column over a sediment half-space. The source is deployed at depth $98 \mathrm{~m}$ and the $30 \mathrm{~cm}$ aperture VSA at $79.9 \mathrm{~m}$ (deepest sensor). The sound speed profile is represented by the dashed line in Fig. 1(right), showing a relatively deep thermocline starting at depth $60 \mathrm{~m}$ giving rise to a narrow acoustic channel bounded by the sea bottom. . The receiver is $1830 \mathrm{~m}$ distant from the source (Fig. 1, left). The bottom is characterized by a compressional speed $c_{s}=1575 \mathrm{~m} / \mathrm{s}$, density $\rho=1.4 \mathrm{~g} / \mathrm{cm}^{3}$, compressional attenuation $\alpha=0.6 \mathrm{~dB} / \lambda$. The source frequency is $13078 \mathrm{kHz}$. The pressure and particle velocity field were synthesized by the TRACEO ray tracing model [17].

It was shown [1], [10] that the vertical particle velocity is the most relevant particle velocity component for bottom (a)

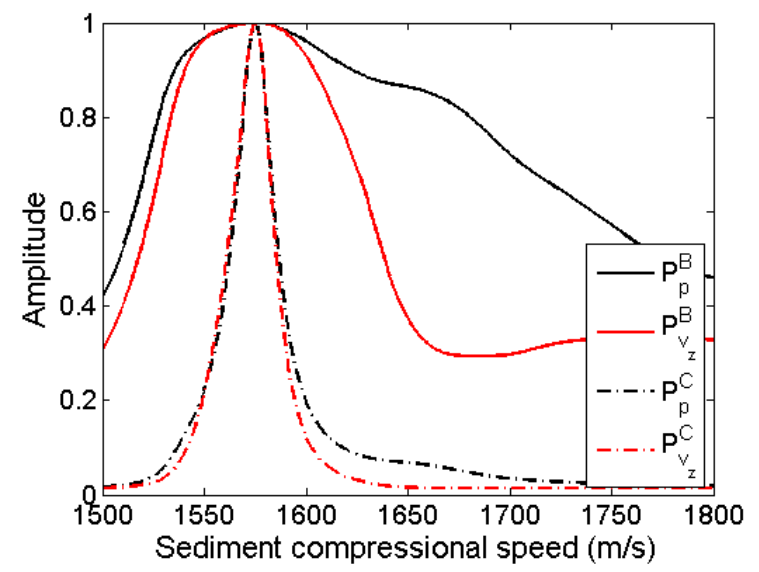

(b)

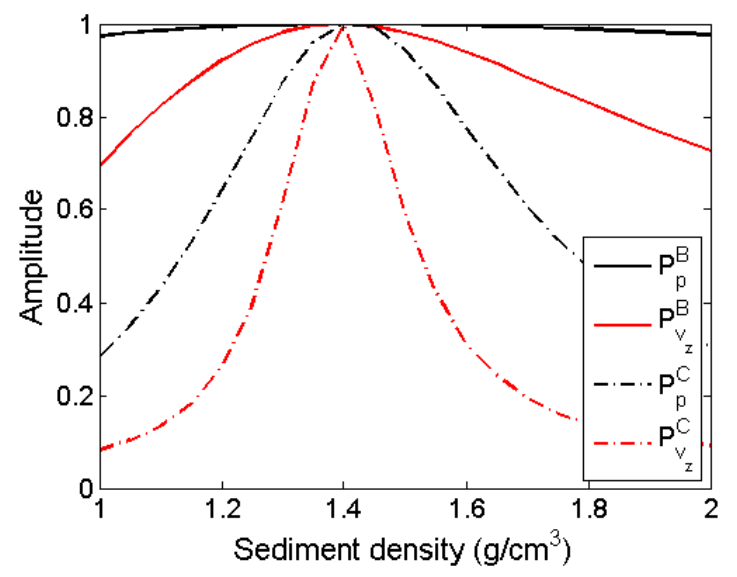

(c)

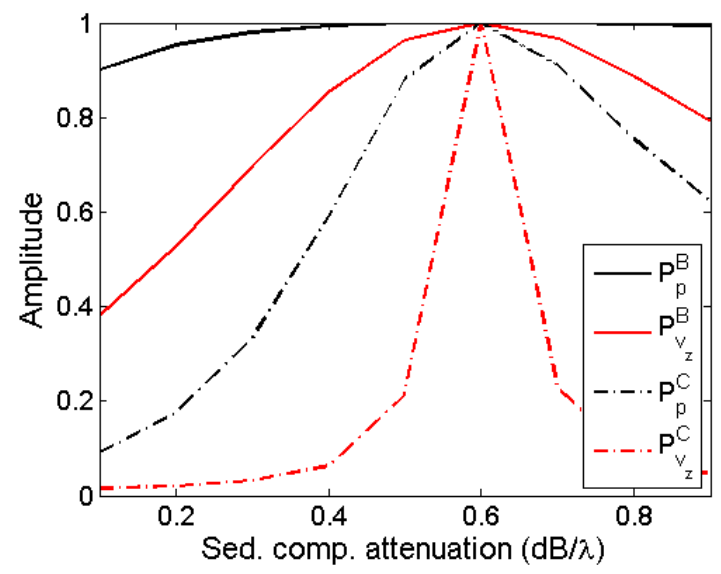

Fig. 2. One-dimensional ambiguity curve obtained with normalized Bartlett (solid) and MVDR (dash-dot) processors at frequency $13078 \mathrm{~Hz}$, for $c_{p}=$ $1575 \mathrm{~m} / \mathrm{s}, \rho=1.4 \mathrm{~g} / \mathrm{cm}^{3}$ and $\alpha=0.6 \mathrm{~dB} / \lambda$ considering the acoustic pressure only (black) and the vertical particle velocity (red) for: sediment compressional speed (a), sediment density (b) and compressional attenuation (c).

characterization. The horizontal components have a behavior similar with pressure. Therefore, this work shows only results 


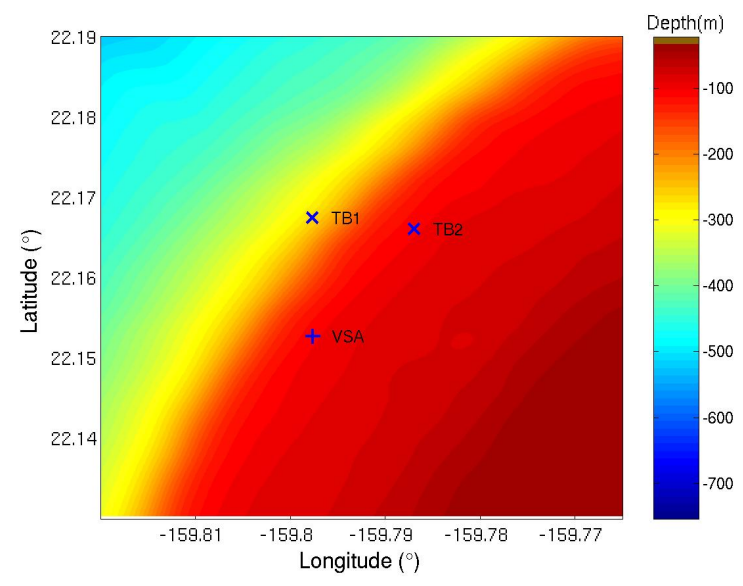

Fig. 3. Makai'05 bathymetry map of the area with the position of the acoustic sources TB1, TB2 and the VSA on September 20th 2005.

from pressure and particle velocity based estimators. The Bartlett and MVDR pressure and vertical particle velocity estimator is determined when the parameters vary in the range: $[1500,1800] \mathrm{m} / \mathrm{s}$ for bottom compressional speed, $[1,2] \mathrm{g} / \mathrm{cm}^{3}$ for density and $[0.1,0.9] \mathrm{dB} / \lambda$ for compressional attenuation. In these simulations it was considered a SNR of $20 \mathrm{~dB}$ and the estimator power was normalized by its overall maximum.

The resulting one-dimensional ambiguity curves for bottom compressional speed, density and compressional attenuation are shown in Fig. 2(a),(b) and (c), respectively, where the pressure related curves are in black and the vertical particle velocity related ones are in red. The solid lines represent Bartlett estimator power, whereas the dash-dotted lines represent MVDR estimator power. It can be seen, both for Bartlett and MVDR estimators, that the main lobe of the particle velocity based estimator for all parameters is significantly narrower (higher parameter resolution) than their pressure only counterparts. When comparing the Bartlett and MVDR estimators, one can notice that even the pressure only MVDR estimator outperforms the vertical particle velocity Bartlett estimator. Under present simulation conditions, the results show that for bottom compressional speed the vertical particle velocity MVDR estimator does not show a significant improvement compared with its pressure counterpart. These simulations suggest that using a MVDR estimator with high frequency signals and a short pressure array, high resolution of the bottom parameter estimates are attained.

\section{EXPERIMENTAL RESULTS}

The data analyzed herein was acquired by a four-element vertical VSA in the $100-14000 \mathrm{~Hz}$ band, during the Makai'05 experiment,off Kauai Island, Hawai (USA), on September 20th, 2005 [9]. The VSA was suspended close to the stern of the R/V Kilo Moana. The probe signals were transmitted from bottom moored acoustic sources, TB1 along a range dependent bathymetry, and TB2 along a range independent bathymetry. The bathymetry map and the location of the equipment are shown in Fig. 3. The geometry between the VSA and the TB2 is similar to that used in simulations (Fig. 1).

The pressure only and vertical particle velocity only Bartlett and MVDR estimators were applied to approximately two hours of a $13078 \mathrm{~Hz}$ tone transmitted from TB2. Results obtained by applying both Bartlett estimators to this data set for the purposes of bottom compressional speed and attenuation, and density were already presented in [1], [18]. The present results addresses the estimation of same set of bottom parameters, but herein the replicas were generated by an updated version of the ray tracing model TRACEO [17] which accounts for shear effects. The replicas used in this work consider a constant shear speed of $67 \mathrm{~m} / \mathrm{s}$ and a shear attenuation of $1.0 \mathrm{~dB} / \lambda$, obtained previously in [19] applying a genetic algorithm based inversion with an extended set of geometric and environmental parameters. Herein, the results were obtained by an exhaustive search for the three bottom parameters.

The estimation results of sediment compressional speed, during this data acquisition period, are shown in Fig. 4. These results were achieved taking into account the most likely values for density and attenuation, $1.35 \mathrm{~g} / \mathrm{cm}^{3}$ and $0.5 \mathrm{~dB} / \lambda$ respectively. Fig. 4 (a) and (b) present the estimation results for sediment compressional speed considering the Bartlett estimator for pressure and vertical particle velocity, respectively, while Fig. 4 (c) and (d) present the estimation results considering the MVDR estimator. It can be seen that the bottom compressional speed results obtained by the pressure estimators are the most stable along time and the MVDR pressure estimator remarkably improves the resolution when compared with its Bartlett equivalent. The vertical particle velocity based estimators presents higher resolution than their pressure counterparts, but on other hand the outliers have an higher impact on the ambiguity surface. It can be seen that the pressure MVDR estimator is the estimator that presents a compromise between stability and resolution.

After the estimation of the sediment compressional speed, the density and the compressional attenuation were estimated taking into account the estimated value of $1535 \mathrm{~m} / \mathrm{s}$ for the sediment compressional speed. Since MFI is less sensitive to such parameters, the results presented in Fig. 5 were achieved considering the geometric mean of the ambiguity surfaces over time, to enhance the best estimation results for both parameters. However, the estimates at instants where bottom sound speed values deviates significantly from its most likely value (outliers) were discarded. Fig. 5 (a) and (b) show the estimation results considering the Bartlett estimator for pressure and vertical particle velocity, respectively, while Fig. 5 (c) and (d) present the estimation results considering the MVDR estimator.

The results show the low sensitivity of the pressure Bartlett estimator to bottom density and compressional attenuation giving rise to a wide main lobe. The pressure MVDR estimator improves significantly the resolution of these parameters, without the trend shown in simulations, where this estimator outperformed vertical particle velocity Bartlett estimator. 
The highest resolution were obtained by the vertical particle velocity estimators, particularly by the MVDR estimator.

Figure 6 presents one dimensional cuts of the bottom compressional speed ambiguity surfaces in Fig. 4 at min. 27 (a) and min. 55 (b), and one dimensional cuts of the bottom density-compressional attenuation ambiguity surfaces in Fig .5 for density (c) and compressional attenuation (d). The peaks of pressure and particle-velocity curves appear at slight different positions. Most likely this difference is due to deviations from the baseline environment on sound speed profile or/and geometric parameters not considered in inversion, which have different impact on the pressure and on the particle velocity field.

\section{CONCLUSION}

This work shows that the MVDR estimator, both for pressure and vertical particle velocity, significantly improves the bottom estimates resolution. Despite the high frequency of the probe signal, the estimates obtained from experimental data using the MVDR estimators, particularly the pressure only, are stable along time. Although, simulation results suggested that pressure MVDR estimator outperforms vertical particle velocity Bartlett estimator, experimental results showed that their resolution are similar, but pressure MVDR estimates were more stable along time. The lower value of bottom compressional speed obtained in this work than previously reported values for the same data set can be ascribed to shear parameters, but this issue requires further investigation. Bearing in mind that actually VS are not widely available, the present work shows that using a MVDR estimator one can attain a sufficient resolution for the bottom parameter estimation, even with a short vertical pressure only array.

\section{ACKNOWLEDGMENT}

The authors would like to thank Michael Porter, chief scientist for the Makai Experiment, Jerry Tarasek for the loan of the vector sensor array used in the experiment, Paul Hursky, Martin Siderius, and Bruce Abraham for providing assistance with the data acquisition. The Makai Experiment was supported by the U.S. Office of Naval Research. This work was funded by National Funds through FCT- Foundation for Science and Technology under project SENSOCEAN (PTDC/EEA-ELC/104561/2008).

\section{REFERENCES}

[1] P. Santos, O. C. Rodriguez, P. Felisberto, and S. M. Jesus, "Seabed geoacoustic characterization with a vector sensor array," Journal of the Acoustical Society of America, vol. 128, no. 5, pp. 2652-2663, November 2010.

[2] P. K. Tam and K. T. Wong, "Cramér-rao bounds for direction finding by an acoustic vector sensor under nonideal gain-phase responses, noncollocation, or nonorthogonal orientation," IEEE Sensors Journal, vol. 9, no. 8, pp. 969-982, August 2009.

[3] M. Hawkes and A. Nehorai, "Wideband source localization using a distributed acoustic vector-sensor array," IEEE Trans. Signal Processing, vol. 27, no. 3, pp. 628-637, July 2002.

[4] J. C. Shipps and B. M. Abraham, "The use of vector sensors for underwater port and waterway security," in Proc. ISA/IEEE Sensors for Industry Conf., August 2004.
[5] A. Song, M. Badiey, P. Hursky, and A. Abdi, "Time reversal receivers for underwater acoustic communication using vector sensors," in Proc. of the OCEANS'08 Conf. Quebec City, Canada: MTS/IEEE, September 2008.

[6] P. Han-Shu and L. Feng-Hua, "Geoacoustic inversion based on a vector hydrophone array," Chinese Physics Letters, vol. 24, no. 7, p. 1977, 2007.

[7] S. Crocker, J. Miller, G. Potty, J. Osler, and P. Hines, "Nonlinear inversion of acoustic scalar and vector field transfer functions," Oceanic Engineering, IEEE Journal of, vol. 37, no. 4, pp. 589-606, 2012.

[8] A. Abdi, H. Guo, and P. Sutthiwan, "A new vector sensor receiver for underwater acoustic communications," in Proc. of the OCEANS'07 Conf. Vancouver, Canada: MTS/IEEE, September 2007.

[9] M. Porter, B. Abraham, M. Badiey, M. Buckingham, T. Folegot, P. Hursky, S. Jesus, K. Kim, B. Kraft, V. McDonald, C. deMoustier, J. Presig, S. Roy, M. Siderius, H. Song, and W. Yang, "The makai experiment: High frequency acoustics," S. M. Jesus and O. C. Rodriguez, Eds., vol. 1, Carvoeiro, Portugal, 2006, pp. 9-18.

[10] P. Felisberto, O. Rodriguez, P. Santos, and S. M. Jesus, "On the usage of the particle velocity field for bottom characterization," in Proc. Int. Conf. on Underwater Acoustic Measurements, Kos, Greece, Kos, Greece, June 2011.

[11] W. A. Kuperman and F. Ingenito, "Spatial correlation of surface generated noise in a stratified ocean," The Journal of the Acoustical Society of America, vol. 67, no. 6, pp. 1988-1996, 1980.

[12] S. Mazzuca, M. Bjork, S. Beer, P. Felisberto, S. Gobert, G. Procaccini, J. Runcie, J. Silva, A. V. Borges, C. Brunet, P. Buapet, W. Champenois, M. M. Costa, D. D’Esposito, M. Gullstrm, P. Lejeune, G. Lepoint, I. Oliv, L. Rasmunsson, J. Richir, M. Ruocco, I. A. Serra, A. Spadafora, and R. Santos, "Establishing research strategies, methodologies and technologies to link genomics and proteomics to seagrass productivity, community metabolism and ecosystem carbon fluxes," Frontiers in Plant Science, vol. 4, no. 38, 2013.

[13] H. Krim and M. Viberg, "Two decades of array signal processing research," IEEE Signal processing magazine, pp. 67-94, July 1996.

[14] M. Hawkes and A. Nehorai, "Acoustic vector-sensor beamforming and capon direction estimation," IEEE Trans. Signal Processing, vol. 46, no. 9, pp. 2291-2304, September 1998.

[15] H.-W. Chen and J.-W. Zhao, "Wideband mvdr beamforming for acoustic vector sensor linear array," Radar, Sonar and Navigation, IEE Proceedings -, vol. 151, no. 3, pp. 158-162, 2004.

[16] D. Johnson and D. E. Dudgeon, Array Signal Processing: Concepts and Techniques. Prentice Hall, Inc.

[17] O. Rodriguez, J. Collis, H. Simpson, E. Ey, J. Schneiderwind, and P. Felisberto, "Seismo-acoustic ray model benchmarking against experimental tank data," Journal of the Acoustical Society of America, vol. 132, no. 2, pp. 709-717, August 2012.

[18] P. Santos, J. Joao, O. C. Rodriguez, P. Felisberto, and S. M. Jesus, "Geometric and seabed parameter estimation using a vector sensor array - Experimental results from Makai experiment 2005," in Proceedings of Oceans 11 IEEE/OES Santander Conference, Santander, Spain, June 6-9 2011.

[19] J. Schneiderwind, Geoacoustic Inversion Applied to the Makai JD264 and JD267 Data, internal rep 01/12 - siplab/cintal ed., CINTAL - Centro Tecnolgico do Algarve, Universidade do Algarve, Campus de Gambelas, 8005-139 Faro, Portugal, October 2012. 
(a)

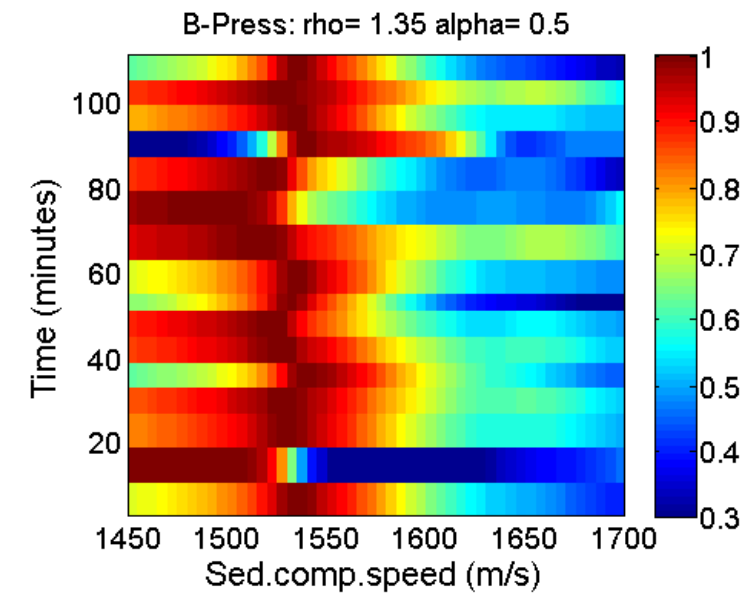

(c)

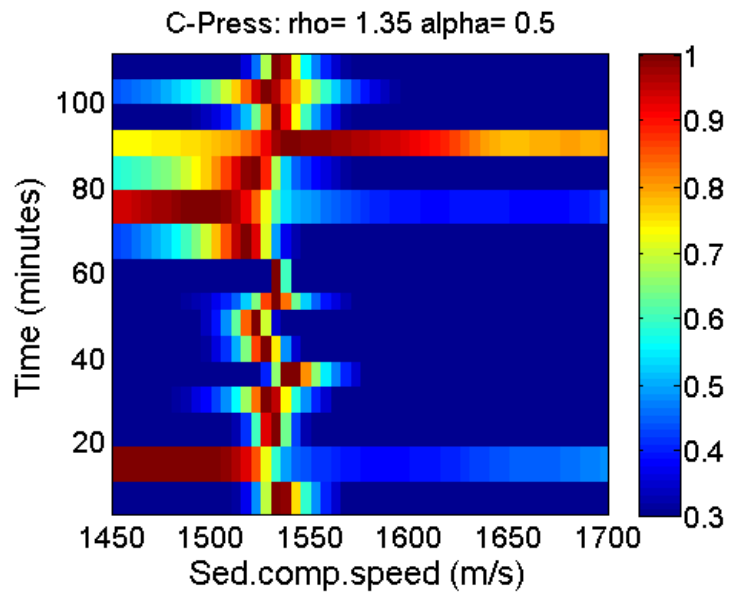

(b)

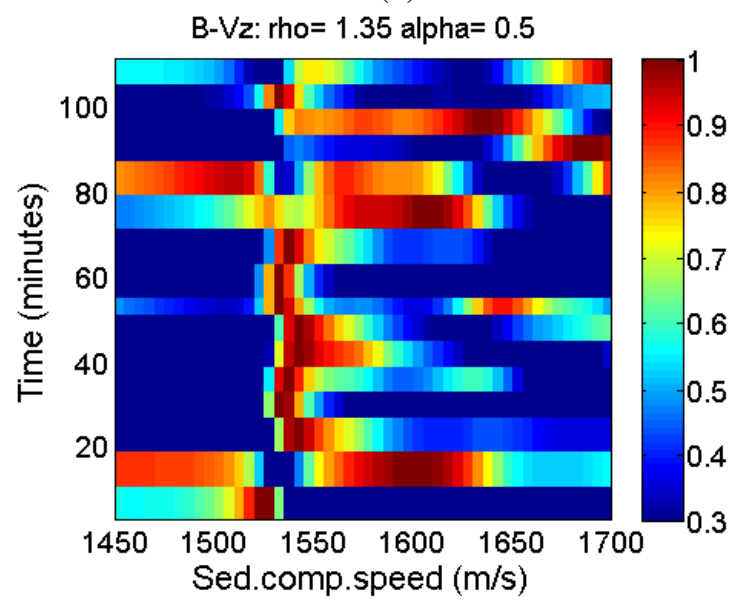

(d)

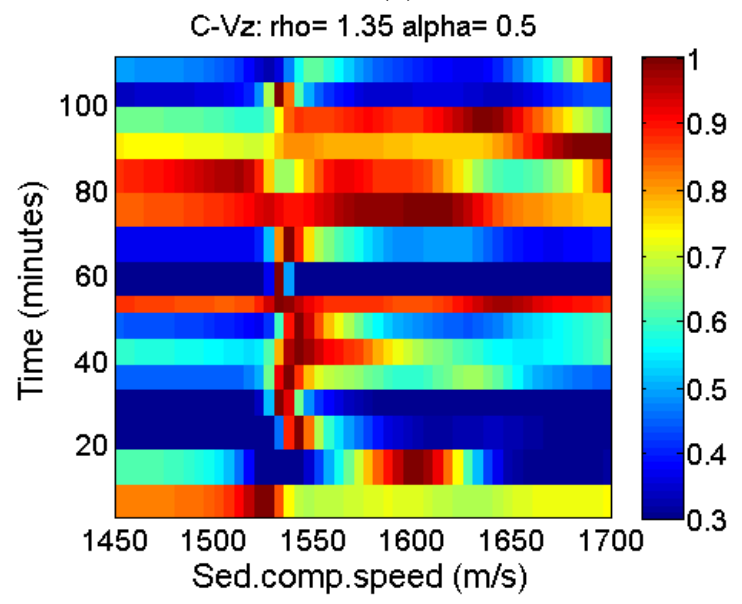

Fig. 4. The experimental data normalized ambiguity surfaces for sediment compressional speed during data acquisition period (two hours), using the Bartlett estimator with pressure (a) and vertical particle velocity (b), and using the MVDR estimator with pressure (c) and vertical particle velocity (d). 
(a)

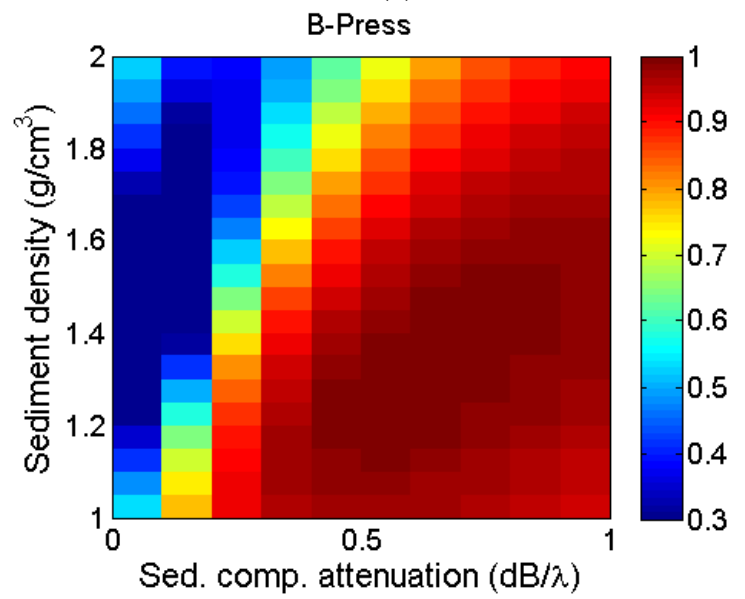

(c)

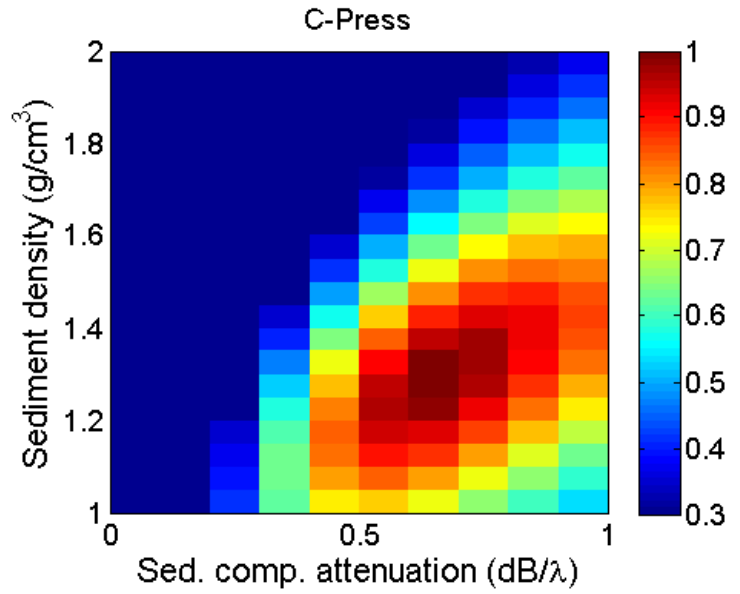

(b)

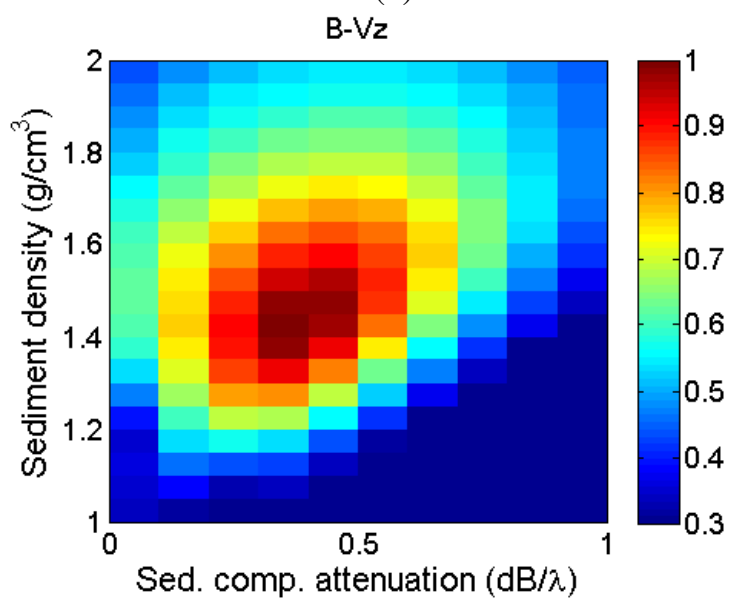

(d)

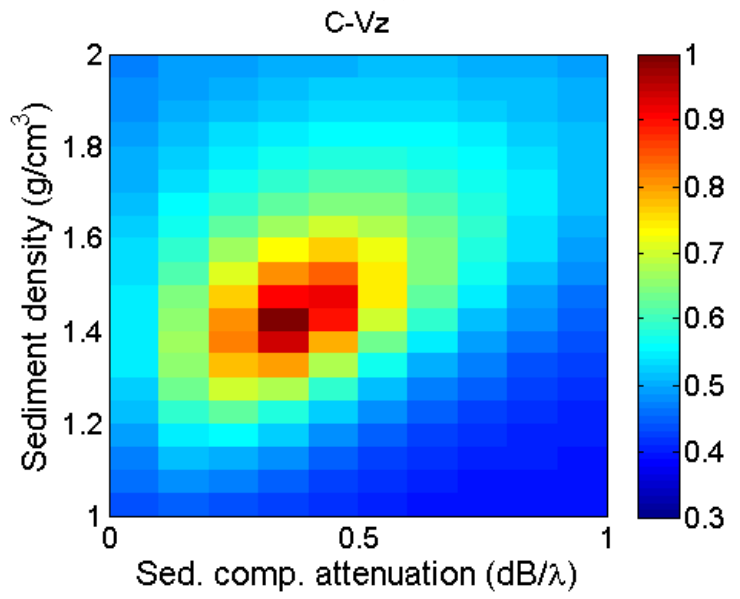

Fig. 5. The experimental data normalized ambiguity surfaces for compressional attenuation and density using the geometric mean over time of estimates along the acquisition period, taking into account the sediment compressional speed of $1535 \mathrm{~m} / \mathrm{s}$ (outliers discarded) considering the Bartlett estimator with pressure (a) and vertical particle velocity (b), and using the MVDR estimator with pressure (c) and vertical particle velocity (d). 
(a)

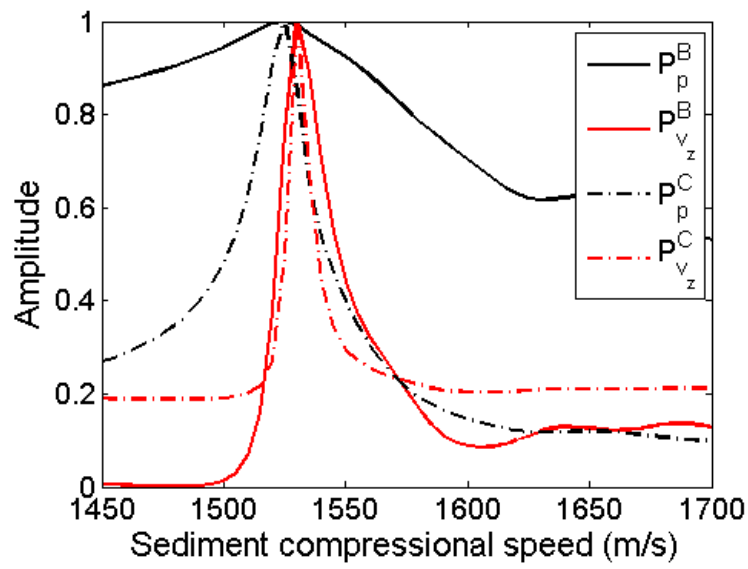

(c)

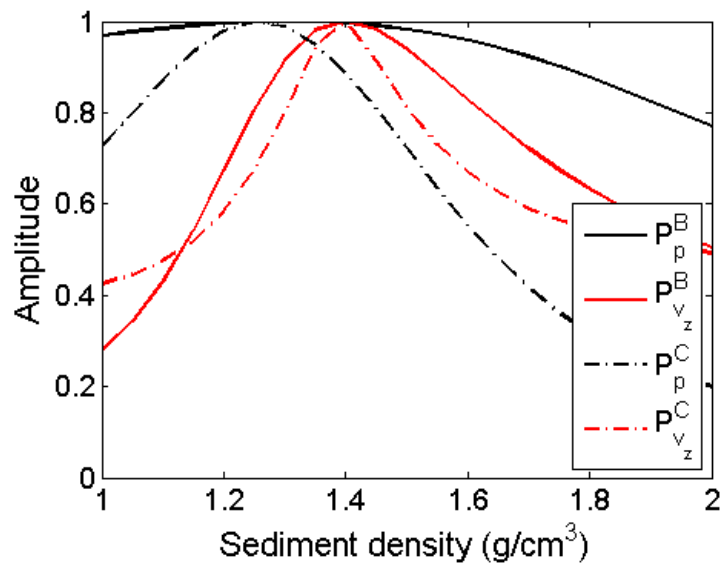

(b)

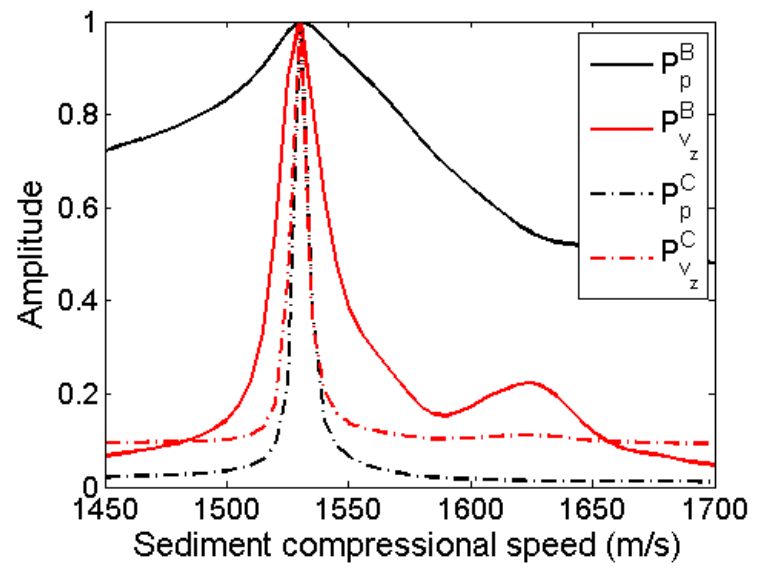

(d)

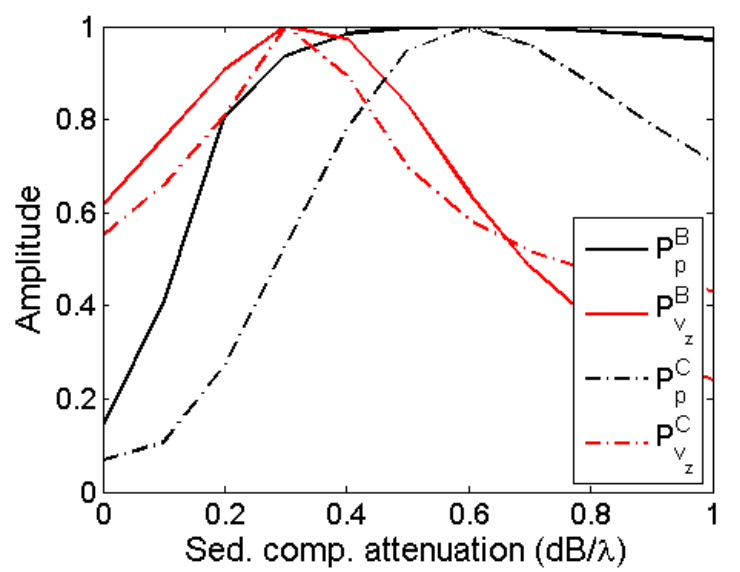

Fig. 6. One-dimensional ambiguity curves (cut) obtained from experimental data presented in Fig. 4 (bottom compressional speed) and Fig. 5 (bottom density and compressional attenuation) for bottom compressional speed at min. 27 (a) and min. 55 (b) and the geometric mean of bottom density (c) and compressional attenuation (d). (Bartlett pressure - solid black, Bartlett vertical particle velocity - solid red, MVDR pressure - dash-dotted black, MVDR vertical particle velocity - dash-dotted red) 